\title{
Two cases report of brachial plexus injury in laparoscopic colorectal surgery
}

\author{
Min-Young No, Jae Moon Shin, and Won-Jun Choi \\ Department of Anesthesiology and Pain Medicine, Sungkyunkwan University School of Medicine, Kangbuk Samsung Hospital, \\ Seoul, Korea
}

The brachial plexus is structurally vulnerable to damage because it is fixed at the cervical vertebra, pre-cervical fascia, and axillary fascia while it extends longitudinally on the surface layer [1]. Causative factors for brachial plexus injury (BPI) during surgery are patients' comorbidity, anatomical malformation, operative factors such as intra-operative postures, operation time, and median sternotomy, and physiological factors such as hypotension and hypothermia. In particular, the Trendelenburg position, which is necessary for laparoscopic and robotic abdominal surgery, may act as a risk factor. We experienced two cases of BPI following colorectal surgery in the Trendelenburg position.

A 34-year-old man underwent laparoscopic total colectomy. In the lithotomy position, his right arm was placed in the neutral position and his left arm was abducted to 90 degrees. During operation, the patient maintained the steep Trendelenburg position, using a shoulder brace, when vital signs were stable. The operative time was 150 minutes, and the anesthesia time was 165 minutes. On Post-operative day (POD) 1, the patient complained of dyskinesia in the left arm and paresthesia in the left arm and hand. The sensory electroneurography (ENG) conducted on POD 11 exhibited decreased amplitude in the left lateral antebrachial cutaneous, radial, and median nerves, and motor ENG revealed decreased amplitude in the left ulna and radial nerves. Also, electromyography (EMG) confirmed damages in the left biceps brachii, pronator teres, pectoralis major, and infraspinatus muscles. Based on such results, he was diagnosed with BPI at the left upper torso or cervical 6 nerve root. Mecobalamine was administered while electrostimulation and kine- siatrics were applied on the left elbow. On POD 28, both motor and sensory functions of the left arm improved. A 52-year-old man underwent hand-assisted laparoscopic anterior resection. He was positioned in the lithotomy position with the right arm in the neutral position and the left arm abducted 90 degrees. The patient was kept in the Trendelenburg position without a shoulder brace. His vital signs were stable. The operative time was 165 minutes, and the anesthesia time was 180 minutes, respectively. After operation, a vestige of being compressed and skin redness on the left shoulder and arm were identified, which were likely to result from the operative bed chafing the left shoulder owing to the Trendelenburg position. On POD 1, the patient complained of dyskinesia in the left shoulder, arm, and hand as well as insensibility in the left lateral forearm. Along with oral administration of mecobalamine and lyrica, electrostimulation and kinesitherapy were performed on weak areas. On POD 7, there were no abnormal findings on the ENG. However, EMG showed that the left deltoid, cervical 7 paraspinal, thoracic 1 paraspinal muscles were damaged. On POD 22, EMG was conducted again to determine the need of medical treatment of damaged muscles and demonstrated considerable improvement. He was then treated with electrostimulation and kinesitherapy for two weeks. On POD 37, the motor function of his left shoulder, arm, and hand and the sensory function of the left lateral forearm were the same as his right side.

In laparoscopy, which has recently become common practice, nerve damages have been reported. Of 2,304 patients who underwent colorectal surgery, the incidence of nerve injury

Corresponding author: Won-Jun Choi, M.D., Department of Anesthesiology and Pain Medicine, Sungkyunkwan University School of Medicine, Kangbuk Samsung Hospital, 108, Pyeong-dong, Jongno-gu, Seoul 110-746, Korea. Tel: 82-2-2001-2001, Fax: 82-2-2001-2326, E-mail: wj2006. choi@samsung.com

(c) This is an open-access article distributed under the terms of the Creative Commons Attribution Non-Commercial License (http:// creativecommons.org/licenses/by-nc/3.0/), which permits unrestricted non-commercial use, distribution, and reproduction in any medium, provided the original work is properly cited. 
including brachial plexus damage was reported to be $3.4 \%$ for laparoscopy patients and $0.2 \%$ for laparotomy patients [2]. The Trendelenburg position is required to secure visibility for laparoscopic abdominal surgery. Shoulder brace and arm board used to immobilize patients may damage the brachial plexus by applying pressure on the acromion and clavicle. In particular, the Trendelenburg position employed with shoulder braces and wrist fixing bands can cause a pulling of the humeral head downward, which may lead to BPI [3].

Our patients experienced BPI even though their laparoscopic colon operation time was relatively short. A difference from the previous cases is whether or not the shoulder brace was used. It is assumed that BPI eventually occurred regardless of the use of shoulder brace or the duration of operation. Putative factors responsible for commonly contributing to BPI in both cases are the Trendelenburg position and changes in the head position and torso position caused by postural lateral movement with the left arm spread.

BPI can be suspected by long operation time, typical symptoms, and physical examination, and can be diagnosed by ENG and EMG. With respect to prognosis, EMG changes appear at 2-3 weeks, so initial EMG is helpful in identifying the degree of existent neurological damages, which generally can be assessed 4 weeks post-injury by re-examination [3]. Physical therapy is the main treatment, while analgesics and non-steroidal antiinflammatory drugs are administered in the case of pain, espe- cially antiepileptic agents such as gabapentin for neuropathic pain [4]. In general, the prognosis of intraoperative BPI is fairly good, and patients mostly should feel symptomatic improvement within 10 weeks [5]. Prevention of BPI primarily requires minimization of operation time and the Trendelenburg position. In addition, hyperextension and flexion to the lateral sides of the head as well as abduction, external rotation, and extension of the arm should be avoided [1]. On this account, the use of arm board and shoulder brace should be restricted, and the neutral position of both arms is recommended. Also, repetitive check on patient's intraoperative postures is necessary for a lengthy operation requiring the steep Trendelenburg position [3]. Recent studies have reported that the utilization of cross chest straps, gel mattress, vacuum bag, etc. was helpful in staving off BPI for long-duration laparoscopy requiring the Trendelenburg position $[2,3]$.

Considering that laparoscopic and robotic abdominal surgery is commonly used, it is naturally expected that the rate of BPI will increase higher than in the past. Now, the patient's posture needs to be considered from the perspectives of not only operative convenience but also of patient safety. Hence, we suggest that anesthesiologists should try to prevent BPI by sharing information about the mechanism, causes, treatment, and prognosis of BPI with attending surgeons and make a prompt recognition of BPI for effective treatment, if any, to be of assistance for intraoperative and intra-anesthetic patient safety.

\section{References}

1. Gagnon J, Poulin EC. Beware of the Trendelenburg position during prolonged laparoscopic procedures. Can J Surg 1993; 36: 505-6.

2. Navarro-Vicente F, García-Granero A, Frasson M, Blanco F, Flor-Lorente B, García-Botello S, et al. Prospective evaluation of intraoperative peripheral nerve injury in colorectal surgery. Colorectal Dis 2012; 14: 382-5.

3. Shveiky D, Aseff JN, Iglesia CB. Brachial plexus injury after laparoscopic and robotic surgery. J Minim Invasive Gynecol 2010; 17: 414-20.

4. Kent CD, Cheney FW. A case of bilateral brachial plexus palsy due to shoulder braces. J Clin Anesth 2007; 19: 482-4.

5. Brill S, Walfisch S. Brachial plexus injury as a complication after colorectal surgery. Tech Coloproctol 2005; 9: 139-41. 